\title{
Palmprint Compression using Wavelet based Contourlet Transform and SPIHT
}

\author{
V. Mohan \\ Associate Professor,Department of ECE, \\ Saranathan College of Engineering, \\ Panjappur, Trichy, Tamil Nadu, India
}

\author{
Y.Venkataramani, Ph. D \\ Professor, Dean (R \& D), \\ Saranathan College of Engineering, \\ Panjappur, Trichy, Tamil Nadu, India
}

\begin{abstract}
Wavelets are capable of approximating images with sharp discontinuities but not good at the approximation of images with smooth contours. Hence contourlet transform was introduced. It produces an efficient multi resolution and directional decomposition of an image using a laplacian pyramidal decomposition and a directional filter bank. It has good approximation capability for smooth $2 \mathrm{D}$ signals. A nonredundant version of this transform, Wavelet Based Contourlet Transform (WBCT) is appropriate for image compression. In this work, the use of WBCT for the compression of palmprint database and its performance are evaluated. The experimental results show that this coding is competitive to the wavelet based compression technique and provides visually superior quality of reconstruction for the mentioned images.
\end{abstract}

\section{Keywords}

Palmprint compression, Wavelet Based Contourlet Transform, Repositioning of WBCT Coefficients, SPIHT encoding of WBCT coefficients.

\section{INTRODUCTION}

Biometric traits such as Iris, Fingerprint, Face and Palmprints are to be compressed using an efficient compression algorithm so that the features of interest should not be degraded. In recent years, several algorithms for compression of biometric image databases are proposed. Different biometric images have different features such as valleys, ridges, rich texture information. So, the compression of such database images requires application-specific algorithms for compression of biometric database images.

In [12], a detailed analysis of all popular lossless image compression algorithms based on transform coding, predictive techniques, dictionary based techniques were carried out. From the results of the experiments it is concluded that the JPEG-LS system is suitable for lossless palmprint compression in terms of its efficiency and storage.

Wavelets are not optimal in capturing the singularities in images. The images which have textures and patterns of oscillatory nature are not effectively represented by wavelets. The smooth contours are also not represented effectively by the wavelet transform. To overcome this, many geometrical transforms have been used in the literature. Since the palmprints consist of ridges, the multi - scale with multiresolution transforms such as

Contourlet transform [1-2] is a multi resolution directional transform. It is capable of capturing contours and fire details in images. It is implemented as a 2 level decomposition. It has good approximation ability for images with smooth contours. Similar to wavelet transform, Contourlet transform has a seamless translation between the continuous and discrete domain representation. The support for $2 \mathrm{~d}$ wavelet basis functions is specified as dyadic squares. Wavelet transform is good for approximating isolated point discontinuities. Contourlet can efficiently extract the directional features with multi- resolution capability from images with textures with smooth contours [1], [2]. Contourlet transform uses a structure similar to that of curvelets. The scheme consists of double filter bank structure, by combining the Laplacian pyramidal decomposition with a directional filter bank [2]. The contourlet transform outperforms the conventional wavelet transforms in terms of capturing the singularities found in the images. In Contourlet transform, a Laplacian pyramidal decomposition of images is implemented in the first stage. The band pass outputs at various levels of Laplacian pyramids are analyzed using Directional Filter Banks (DFB) for extracting the angular information [5]. But due to redundancy nature of Laplacian pyramidal representation of images, the use of contourlet transform has not become popular for image compression algorithms [6-7].

In order to have a non redundant contourlet transform, the octave band directional filter banks [3], the Critically Sampled Contourlet (CRISP) Transform [4] and the wavelet-based Contourlet Transform [6] are proposed. In [3], Hong and smith proposed the octave band directional filter banks which are capable of performing the decomposition of image data into angular directional and octave band radial details. In [4], $\mathrm{Lu}$ and Do proposed a single stage implementation of a non separable filter bank based critically sampled contourlet transform to provide a non-redundant directional decomposition. In this work, the palmprint compression using Wavelet- Based Contourlet Transform has been implemented which is a multi scale, multi - directional transform with modified SPIHT coder. 


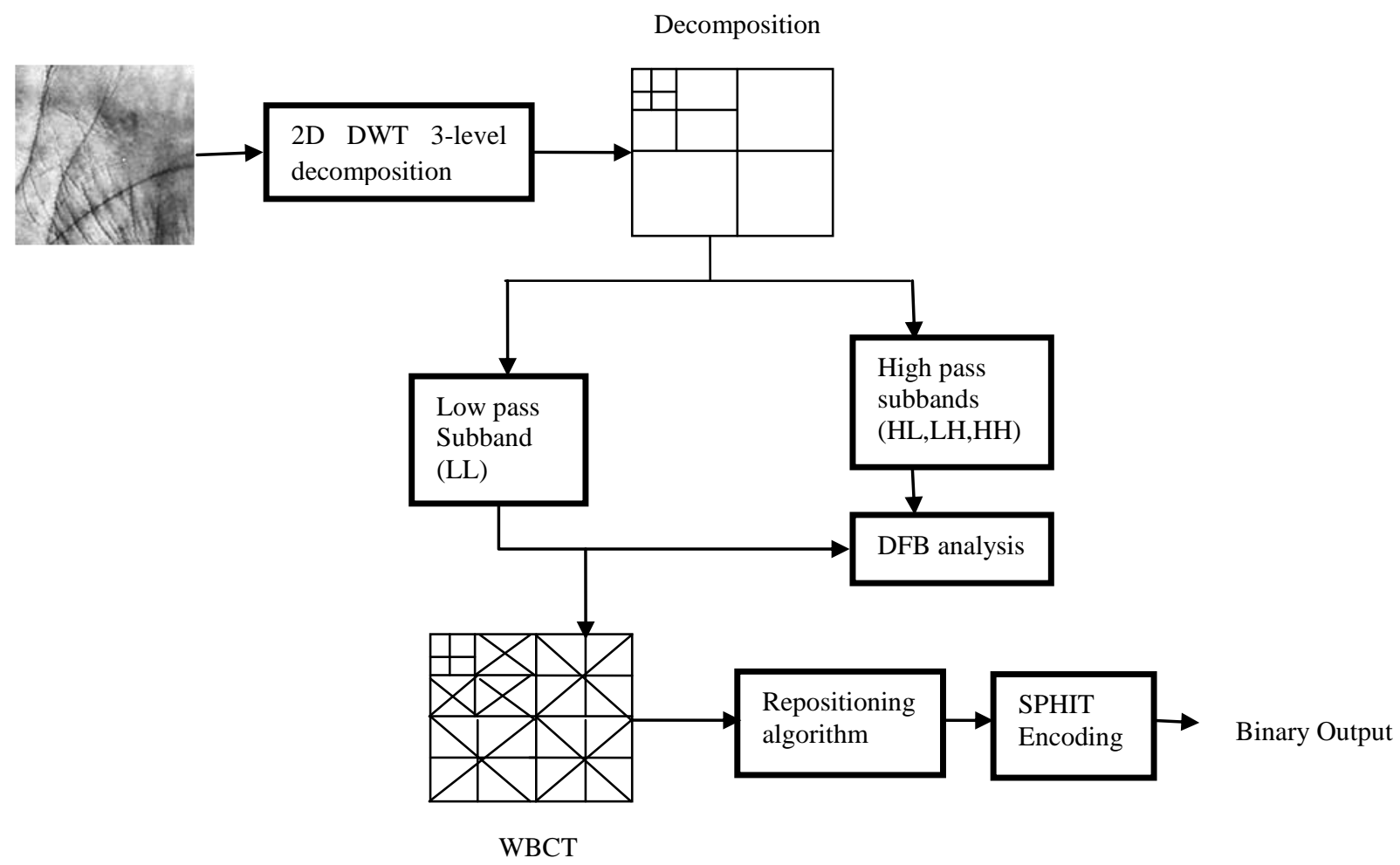

Fig. 1 Flow Diagram of the proposed scheme using Wavelet Based Directional Transform

It is inspired by the work proposed in [6]. The WBCT is implemented by directional filter bank analysis of wavelet subbands. The parent -children relationship between coefficients in different subbands in WBDT domain is different from that of wavelet domain. In order to use the encoding algorithms designed for wavelet domain, a repositioning algorithm [6] for WBDT coefficients is implemented. Repositioning algorithm is useful in generating spatial orientation trees similar to the wavelet tree structure. The rearranged coefficients are encoded using SPIHT encoder used for Wavelet decomposition.

The rest of the paper has been organized as follows. Section 2 describes the theoretical backgrounds of WBDT and the construction of DFB. Section 3 explains the WBDT based compression scheme with a rearrangement algorithm. Section 4 illustrates the implementation of the proposed palmprint coder with the simulation results.

\section{WAVELET BASED DIRECTIONAL TRANSFORM}

Eslami and Radha [6] proposed a new family of geometrical image transform that decomposes image to be compressed both radial and angularly. Its salient feature is that it is non redundant and it has the ability of perfect reconstruction. The first stage provides subband decomposition, which in the case of the WBCT is a wavelet transform, in contrast to the Laplacian pyramid used in contourlets. The second stage of the WBCT is a directional filter bank (DFB), which provides angular decomposition. The first stage is realized by separable filter banks, while the second stage is implemented using nonseparable filter banks. For the DFB stage, the iterated treestructured filter banks using fan filters [2] are constructed.

Wavelet coefficients are further analyzed to decompose them with directional filter banks. The input image is split into various subbands using analysis filter banks and wavelet coefficients are calculated. The analysis directional filter banks are used to decompose all the high frequency subbands into angular subbands. The wavelet decomposition followed by DFB analysis is called Wavelet Based Contourlet Transform of an image.

At each level $(j)$ in the wavelet transform, three highpass bands corresponding to the $\mathrm{LH}, \mathrm{HL}$, and $\mathrm{HH}$ bands are obtained. Non redundant DFB analysis is carried out with the same number of directions to each band in a given level $(j)$. Starting from the maximum number of desired directions $2^{L}$ on the finest level $J$, of the wavelet transform, the number of directions at every other dyadic scale is decreased through the coarser levels $(j<J)$ which satisfies the anisotropy scaling law; that is width $\approx$ length ${ }^{2}$.

Since mostly vertical directions exist in the HL subband and horizontal directions are found in the LH image, it might seem logical to use partially decomposed DFBs with vertical and horizontal directions on the HL and LH bands, respectively. However, since the wavelet filters are not perfect in splitting the frequency space to the lowpass and highpass components, that is, not all of the directions in the HL image are vertical and in the LH image are horizontal, further decomposition using DFB is carried out on each subband.

Similar to the Contourlet transform, the WBDT consists of two filter bank stages. The flow diagram is in fig 1 . The first stage provides subband decomposition which, in the case of WBDT, is a wavelet transform. The Mallat's Pyramidal decomposition is used in contrast to the Laplacian Pyramid used in Contourlets. The second stage of the WBDT is the Directional filter bank (DFB) analysis which provides angular decomposition [8]. For the DFB stage, the iterated tree structure filter banks using fan filters are employed. DFB with equal number of directional decompositions to each high pass band at that level of subband decomposition are applied. At nth level decomposition in the wavelet transform, the LH, HL and $\mathrm{HH}$ detail subbands are produced. Angular analysis of 
wavelet subbands is carried out using DFB with the same number of directions to each subband at the specified level. Direction analysis with maximum number of directions is performed on the wavelet subbands of the finest level. The number of directions for the next consecutive level subband is decreased so as to satisfy anisotropy scaling law.

Bamberger et al. [10] presented a minimally decimated initially sampled 2-D DFB along with a proposal for perfect reconstruction. The DFB proposed by Bamberger et al. is a single level tree structured Filter Bank which is used to decompose the images into number of directions. The DFB construction explained in the thesis of Do [1] uses diamond shaped and fan - beam filters in the Quincnux Filter Bank(QFB) [10]. The filters in the DFB are designed to capture the directional high frequency features of the image. The DFB alone cannot provide a sparse representation of the image. It is always combined with multi-resolution analysis system. The high pass subbands generated by the Mallat's wavelet decomposition scheme are analyzed using directional filter bank at different scales.

\section{REARRANGEMENT OF COEFFICIENTS AND ENCODING}

Repositioning of children coefficients is done to cluster the children wavelet coefficients which are repositioned because of the further DFB analysis of wavelet subbands. To partition and refine the significant coefficients in the subbands of WBCT, three sets of LIP (list of insignificant pixels), LIS (List of insignificant sets), and LSP (list of significant pixels) are generated from the subbands as it is specified in the SPIHT coding of wavelet subband coefficients. A spatial orientation tree or a zero tree is constructed from the wavelet coefficients across different subbands at different scales based on the parent child relationship between coefficients [9]. In WBCT decomposition, the scale in which the children coefficients will be twice as that of directional subbands in which the parent coefficients are located. The subbands in which the children are available are generated from the parent directional subband by the directional decomposition using directional filter banks. Because of the differences in the positions of parent - children coefficients and their structures between wavelet subbands and the wavelet based subbands, it is necessary to reposition and merge the subbands in which the children coefficients are located in the WBCT structure before encoding it using the SPIHT [8]. The repositioned coefficients are encoded with SPIHT encoder. Thus a compressed form of input image is obtained.

In the receiver, first decoded using a SPIHT decoder and then the repositioned subbands are arranged back into a structure similar to the WBCT decomposition. Once the subband structure similar to the WBCT structure is created from decoded subbands, the synthesis DFB followed by synthesis wavelet filters are used to reconstruct the image from the decoded coefficients.

\section{IMPLEMENTATION}

The proposed WBCT/SPIHT image coder for palmprint compression is implemented using Matlab. The palmprints are resized to the size of $512 \times 512$ pixels. The filters chosen for the two stages of decomposition are 9/7 filter and PKVA filters. The $9 / 7$ filter is a bi-orthogonal filter and PKVA filter is coined from the first names of the four authors who proposed it, Phoong, Kim, Vaidyanathan, and Ansari [7] . It is of quincunx/fan filter type. The input palmprint is decomposed using 2D wavelet transform through $9 / 7$ filterbank. For contoured images, Biorthogonal wavelet filterbank yields good subband decomposition. Three levels subband analysis is carried out. So, totally 10 subbands are generated. Each high frequency band at various resolution are further decomposed using dfbdec( ) - matlab function which is available in the controulet toolbox. The contourlet toolbox is useful for performing directional analysis of input images. Among the three level wavelet decompositions, the finer level decomposition has 8 level directional decompositions and the second level uses 4 level directional decompositions and the coarser level uses no directional decomposition. The coefficients of Wavelet Based Contourlet Transformed image are arranged such that the arrangements of coefficients are identical to the arrangement of wavelet subband decompositions.

The WBCT coefficients are scanned using set partitioning in Hierarchical Trees scanning and encoding procedure. The program is written such that the output of the SPIHT program for WBCT will be a binary output sequence to indicate the significance of the WBCT at each scanning level.

The performances are tabulated in Table-I and Table -II and also represented as graphs in fig. 3 and fig. 4

From the experimental results, it is evident that the performance of WBCT is superior to that of the wavelet transform based compression techniques both in terms PSNR in $\mathrm{dB}$ versus rate in bpp and visual quality o f reconstruction

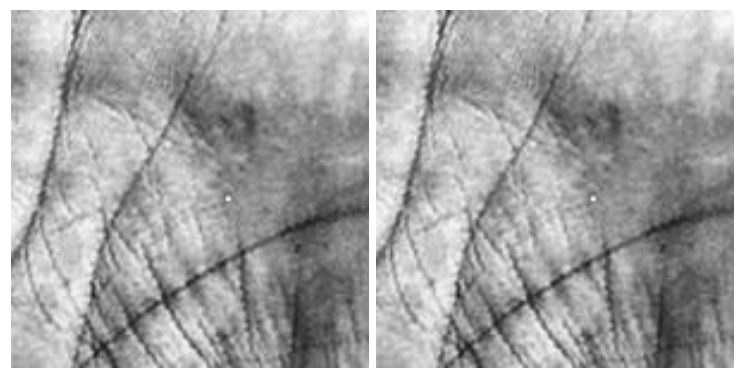

\section{Fig 2 (a) Input palmprint (b) Reconstructed Image using} WBDT and SPIHT algorithm

The quality of reconstruction in terms of PSNR in $\mathrm{dB}$ for palmprint-A using wavelet transform based SPIHT are given in Table II. From the Table II, it is evident that as the level of decomposition increases, the quality of reconstruction in terms of PSNR in $\mathrm{dB}$ also increases. There is roughly about 3 $\mathrm{dB}$ increase in PSNR for the same compression ratio available at lower decomposition level. The results for WT-SPIHT and WBDT-SPIHT are compared and the comparative results are also tabulated in Table I. This implies that the user can get extra quality at the reconstruction end using WBDT - SPIHT. The reconstruct ed palmprint-A using both WT-SPIHT and WBDT-SPIHT are shown in Fig. 4. 


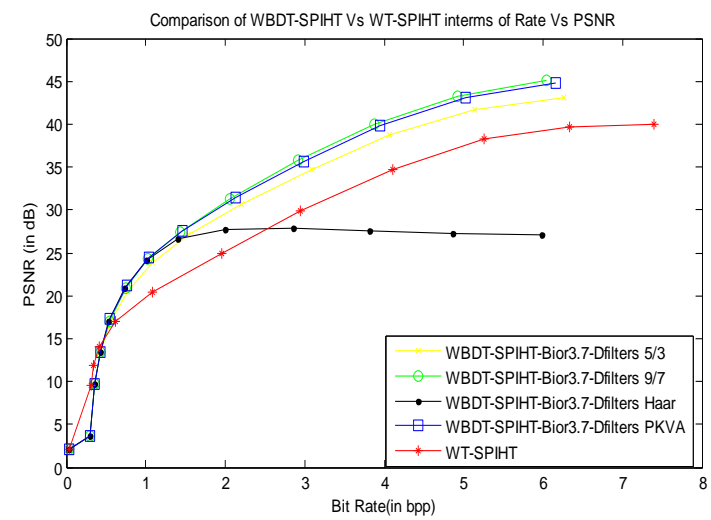

Fig. 3 Comparison of WBDT-SPIHT and WT-SPIHT

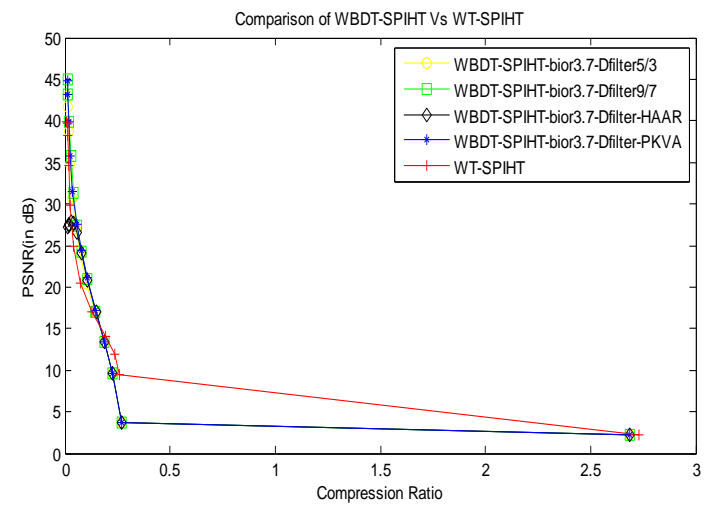

Fig. 4 Comparison of WBDT-SPIHT and WT-SPIHT in terms of PSNR (dB) Vs Compression Ratio

Table I Comparison of Rate Vs PSNR for various levels decomposition

\begin{tabular}{|c|c|c|c|c|c|c|c|c|c|c|}
\hline \multirow{3}{*}{$\begin{array}{l}\text { Rate } \\
\text { (bpp) }\end{array}$} & \multicolumn{8}{|c|}{ PSNR in $\mathrm{dB}$ for the reconstructed images using WBDT- SPIHT } & \multirow{2}{*}{\multicolumn{2}{|c|}{$\begin{array}{c}\text { Rate in bpp Vs } \\
\text { PSNR in dB } \\
\text { using Wavelet + } \\
\text { SPIHT }\end{array}$}} \\
\hline & \multicolumn{2}{|c|}{$\begin{array}{c}\text { WBDT- SPIHT WT } \\
\text { filter-bior3.7 } \\
\text { D-filter-5/3 } \\
\end{array}$} & \multicolumn{2}{|c|}{$\begin{array}{c}\text { WBDT- SPIHT } \\
\text { WT filter-bior3.7 } \\
\text { D-filter-9/7 }\end{array}$} & \multicolumn{2}{|c|}{$\begin{array}{l}\text { WBDT- SPIHT } \\
\text { WT filter-bior3.7 } \\
\text { D-filter-HAAR }\end{array}$} & \multicolumn{2}{|c|}{$\begin{array}{c}\text { WBDT- SPIHT } \\
\text { WT filter-bior3.7 } \\
\text { D-filter-PKVA }\end{array}$} & & \\
\hline & $\mathrm{CR}$ & PSNR & $\mathrm{CR}$ & PSNR & $\mathrm{CR}$ & PSNR & $\mathrm{CR}$ & PSNR & $\mathrm{CR}$ & PSNR \\
\hline 0.03 & 2.69 & 2.15 & 2.69 & 2.15 & 2.69 & 2.15 & 2.69 & 2.15 & 2.73 & 2.15 \\
\hline 0.30 & 0.27 & 3.64 & 0.27 & 3.64 & 0.27 & 3.64 & 0.27 & 3.64 & 0.26 & 9.47 \\
\hline 0.35 & 0.23 & 9.69 & 0.23 & 9.69 & 0.23 & 9.69 & 0.23 & 9.69 & 0.23 & 11.86 \\
\hline 0.43 & 0.19 & 13.45 & 0.19 & 13.45 & 0.19 & 13.45 & 0.19 & 13.45 & 0.19 & 14.06 \\
\hline 0.56 & 0.14 & 16.94 & 0.15 & 17.06 & 0.15 & 17.01 & 0.15 & 17.27 & 0.13 & 17.03 \\
\hline 0.78 & 0.10 & 20.56 & 0.11 & 20.95 & 0.11 & 20.82 & 0.11 & 21.16 & 0.07 & 20.44 \\
\hline 1.08 & 0.07 & 23.90 & 0.08 & 24.28 & 0.08 & 24.12 & 0.08 & 24.41 & 0.04 & 25.00 \\
\hline 1.53 & 0.05 & 27.02 & 0.06 & 27.46 & 0.06 & 26.59 & 0.05 & 27.62 & 0.03 & 29.87 \\
\hline 2.21 & 0.04 & 30.59 & 0.04 & 31.29 & 0.04 & 27.72 & 0.04 & 31.45 & 0.02 & 34.69 \\
\hline 3.09 & 0.03 & 34.77 & 0.03 & 35.77 & 0.03 & 27.80 & 0.03 & 35.71 & 0.02 & 38.25 \\
\hline 4.07 & 0.02 & 38.78 & 0.02 & 39.96 & 0.02 & 27.54 & 0.02 & 39.84 & 0.01 & 39.67 \\
\hline 5.14 & 0.02 & 41.66 & 0.02 & 43.21 & 0.02 & 27.32 & 0.02 & 43.12 & 0.01 & 39.96 \\
\hline 6.26 & 0.01 & 43.06 & 0.01 & 45.04 & 0.01 & 27.16 & 0.01 & 44.86 & 0.01 & 40.01 \\
\hline
\end{tabular}


Table II Comparison of Rate Vs PSNR for various levels decomposition using WT-SPIHT

\begin{tabular}{|c|c|c|c|c|c|c|c|c|c|c|c|}
\hline \multicolumn{12}{|c|}{ PSNR in dB and CR using Wavelet based Contourlet Transform + SPHIT } \\
\hline Level & $\begin{array}{c}\text { Rate } \\
\text { (bpp) }\end{array}$ & $\begin{array}{c}\text { Compressi } \\
\text { on Ratio }\end{array}$ & $\begin{array}{l}\text { PSNR } \\
\text { (in dB) }\end{array}$ & Level & $\begin{array}{l}\text { Rate } \\
\text { (bpp) }\end{array}$ & $\begin{array}{l}\text { Compress } \\
\text { ion Ratio }\end{array}$ & $\begin{array}{l}\text { PSNR } \\
\text { (in dB) }\end{array}$ & Level & $\begin{array}{c}\text { Rate } \\
\text { (bpp) }\end{array}$ & $\begin{array}{c}\text { Compr } \\
\text { ession } \\
\text { Ratio }\end{array}$ & $\begin{array}{l}\text { PSNR } \\
\text { (in } \\
\text { dB) }\end{array}$ \\
\hline \multirow{13}{*}{2} & 0.03 & 2.69 & 2.15 & \multirow{13}{*}{3} & 0.03 & 2.73 & 2.15 & \multirow{13}{*}{4} & 0.03 & 2.69 & 2.15 \\
\hline & 1.16 & 0.07 & 8.86 & & 0.30 & 0.26 & 9.47 & & 0.10 & 0.82 & 9.51 \\
\hline & 1.25 & 0.06 & 13.33 & & 0.35 & 0.23 & 11.86 & & 0.14 & 0.57 & 11.40 \\
\hline & 1.45 & 0.06 & 16.86 & & 0.43 & 0.19 & 14.06 & & 0.18 & 0.44 & 12.82 \\
\hline & 1.92 & 0.04 & 20.36 & & 0.56 & 0.13 & 17.03 & & 0.25 & 0.32 & 14.30 \\
\hline & 2.78 & 0.03 & 25.00 & & 0.78 & 0.07 & 20.44 & & 0.46 & 0.18 & 17.10 \\
\hline & 3.79 & 0.02 & 30.00 & & 1.08 & 0.04 & 25.00 & & 0.92 & 0.09 & 20.47 \\
\hline & 4.96 & 0.02 & 35.12 & & 1.53 & 0.03 & 29.87 & & 1.80 & 0.04 & 25.00 \\
\hline & 6.13 & 0.01 & 39.30 & & 2.21 & 0.02 & 34.69 & & 2.78 & 0.03 & 29.88 \\
\hline & 7.23 & 0.01 & 41.17 & & 3.09 & 0.02 & 38.25 & & 3.94 & 0.02 & 34.69 \\
\hline & 8.30 & 0.01 & 41.58 & & 4.07 & 0.01 & 39.67 & & 5.10 & 0.02 & 38.25 \\
\hline & 8.34 & 0.01 & 41.75 & & 5.14 & 0.01 & 39.96 & & 6.17 & 0.01 & 39.66 \\
\hline & 8.35 & 0.01 & 42.01 & & 6.26 & 0.01 & 40.01 & & 7.23 & 0.01 & 39.96 \\
\hline
\end{tabular}

\section{REFERENCES}

[1] M. N. Do, Directional multiresolution image representations. $\mathrm{PhD}$ thesis, EPFL, Lausanne, Switzerland, Dec. 2001.

[2] M. N. Do and M. Vetterli, "Contourlets in Beyond Wavelets”, Academic Press, New York, 2003.

[3] P. Hong and M. J. T. Smith, "An octave-band family of nonredundant directional filter banks", IEEE proceedings of ICASSP, vol. 2, pp.1165-1168, 2002.

[4] Y. Lu and M. N. Do, "CRISP-contourlets: a critically sampled directional multiresolution image representation," in proc. of SPIE conference on Wavelet Applications in Signal and Image Processing $X$, San Diego, USA, August 2003.

[5] Arthur L. da Cunha, Jianping Zho and Minh N. Do, "The Nonsubsampled Contourlet Transform: Theory, Design and Applications", IEEE Transactions on Image Processing, Vol. 15, No.10, pp. 3089 - 3101, Oct. 2006.

[6] Ramin Eslami, Hayder Radha, "Wavelet-Based Contourlet Transform and its Application to Image Coding", IEEE International Conference on Image Processing (ICIP 2004), Vol. 5, pp. 3189-3192, Oct. 2004.
[7] S. M. Phoong, C. W. Kim, P. P. Vaidyanathan, and R. Ansari, "A new class of two- channel biorthogonal filter banks and wavelet bases," IEEE trans. on SP, vol. 43, no. 3, pp. 649-665, Mar. 1995.

[8] R. Sudhakar, R. Karthiga, and S. Jayaraman, "Fingerprint Compression Using Contourlet Transform with Modified SPIHT Algorithm", Iranian Journal Of Electrical And Computer Engineering, Vol. 5, No. 1, Winter-Spring 2006.

[9] Said and W. A. Pearlman, "A New Fast and Efficient Image Codec Based on Set Partitioning in Hierarchical Trees," IEEE Trans. on Circuits and Systems for Video Technology, vol. 6, pp. 243-250,June, 1996.

[10] R. H. Bamberger, M. J. T. Smith, “A filter bank for the directional decomposition of images: Theory and design”, IEEE Trans. Signal Processing, vol. 40, no. 4, pp.-882-893, April 1992.

[11] Song Zhao, Yan Xu,Hengjian Li, and Heng Yang, “A Comparison of Lossless coompression Methods for Palmprint Images", Journal of Software, Vol. 7, No. 3, March

2012 